\title{
Rehabilitación vestibular de la Enfermedad de Meniere en el estadio tardío. Reporte de un
}

\section{Caso}

\author{
Vestibular rehabilitation of Meniere's Disease in its chronic stage. A case report \\ Yuly Polo-Espinoza 1,a, Martín J. Previgliano ${ }^{2, b}$, Janet Jara-López ${ }^{1, a}$, Lizbeth Ramírez-García ${ }^{1, a}$, \\ Raquel Tapia-Egoavil ${ }^{1, c}$
}

\section{RESUMEN}

La enfermedad de Meniere (EM), se caracteriza por ataques fluctuantes de vértigos, hipoacusia y acúfenos. Una vez que se controlan los síntomas agudos, la rehabilitación vestibular es una alternativa de tratamiento efectiva. Se presenta el caso de una mujer de 46 años con EM en la fase crónica que presenta persistencia de vértigo postural e inestabilidad de la marcha, quien fue intervenida con maniobras de reposición y rehabilitación vestibular con ejercicios de adaptación y sustitución, ejercicios de habituación del equilibrio vestibular y de control postural. Se obtuvo una respuesta favorable, con desaparición del vértigo posicional, disminución significativa de la inestabilidad y gran mejoría en su calidad de vida. No se puede llegar a conclusiones con un solo caso, sin embargo, es importante el abordaje integral en el tratamiento de pacientes con EM.

PALABRAS CLAVE: Rehabilitación, vestíbulo del laberinto, enfermedad de Meniere. (Fuente: DeCS BIREME).

\section{SUMMARY}

Meniere's disease presents fluctuating attacks of dizziness, hearing loss and tinnitus. Once acute symptoms are controlled, vestibular rehabilitation is an effective treatment alternative. We present the case of a 46-year-old woman with Meniere's disease in the chronic phase, who presented persistent postural vertigo and gait instability and who was treated with repositioning maneuvers, along with vestibular rehabilitation with adaptation, substitution and habituation exercises, vestibular balance and postural control. A favorable response is achieved, with the disappearance of positional vertigo, a significant decrease in instability and a great improvement in her quality of life. No conclusions can be drawn from a single case; however, a comprehensive approach is important in the treatment of patients with MS.

KEYWORDS: Rehabilitation, vestibule, labyrinth, Meniere disease. (Source: MeSH NLM).

1 Departamento de Investigación, Docencia y Rehabilitación Integral en Amputados, quemados y Trastornos Posturales, Instituto Nacional de Rehabilitación Dra. Adriana Rebaza Flores Amistad Perú-Japón. Chorrillos, Perú.

2. Centro Martin Previgliano. Buenos Aires, Argentina.

3. Tecnólogo Médico en el área Terapia Física y Rehabilitación;

4. Lic. Kinesiólogo Fisiatra;

5. Médico Rehabilitador. 


\section{INTRODUCCIÓN}

La enfermedad de Meniere (EM), cursa con un cuadro discapacitante de ataques episódicos de vértigo, hipoacusia y acúfenos (1); en su evolución al estadio tardío, los episodios de vértigo disminuyen y desaparecen, pero se va instaurando el desequilibrio permanente (2).

Existe una asociación de la EM con el vértigo posicional paroxístico benigno (VPPB), que varía de $10 \%$ a $32,8 \%(2,3)$; y en este contexto, los pacientes refieren sensación de vértigo con los cambios posturales. El VPPB, puede preceder al desarrollo de la EM o se presenta en el curso de la EM (2).

Las crisis de vértigo de la EM, limitan las actividades ante el temor de enfrentarse a circunstancias sociales y otras situaciones; aún sean leves, el paciente presenta conductas de aislamiento (4).

La edad de inicio es entre la tercera y cuarta década, con presentación similar entre hombres y mujeres; las prevalencias son muy variadas, debido a diversos criterios usados en el diagnóstico (1), en un estudio realizado en Finlandia, se encontró una prevalencia de $513 / 100000(5)$.

La rehabilitación vestibular (RV) en la EM promueve la compensación vestibular y reduce los síntomas, en el estadio inicial los resultados son limitados por la disfunción vestibular fluctuante. Por el contrario, la RV es eficaz cuando por la evolución de la enfermedad o por un tratamiento, se ha convertido en una disfunción vestibular permanente y en el que se establece un cuadro de inestabilidad o desequilibrio (2).

La RV es efectiva y muy recomendada en los pacientes con EM, lográndose una mejora significativa en la función del equilibrio (6), tiene como componentes la valoración, educación y ejercicios de adaptación para crear nuevos procesos funcionales $(7,8)$, incluye ejercicios de sustitución para recuperar la función perdida con nuevas estrategias, ejercicios de habituación para lograr un cambio en la respuesta sintomática al estímulo después de la repetición, ejercicios de reeducación del equilibrio, de la marcha y maniobras de reposición para lograr respuestas compensatorias frente a los vértigos posicionales (4). Además, se complementa con terapia kinésica de reacondicionamiento físico, relajación, respiración, y corrección de alteraciones posturales (8).

El objetivo de este reporte fue mostrar los efectos beneficiosos de la RV en una paciente con persistencia de vértigo postural e inestabilidad de la marcha por EM en el estadio tardío.

\section{PRESENTACIÓN DEL CASO}

Mujer de 46 años, sin antecedentes de importancia, con historia de vértigos de 20 años de evolución que se exacerbaron de forma progresiva, tinnitus unilateral

Tabla 1. Resultados clínicos de la intervención con rehabilitación vestibular.

\begin{tabular}{lcc}
\hline Evaluación & $\begin{array}{c}\text { Antes de la intervención con } \\
\text { Rehabilitación Vestibular }\end{array}$ & $\begin{array}{c}\text { Después de } \mathbf{2} \text { meses } \\
\text { de la intervención con } \\
\text { Rehabilitación Vestibular }\end{array}$ \\
\hline Vértigo posicional & Positivo & Negativo \\
Reflejo vestíbulo-ocular lento & Alterado & Negativo \\
Reflejo vestíbulo-ocular rápido & Alterado & Alterado \\
Prueba de Barany & Positivo & Negativo \\
Test posicional de Dix- Hallpike & Positivo & Negativo \\
Prueba de Romberg & Positivo (+++) & Positivo (+) \\
Prueba de Romberg sensibilizado (Tandem) & Positivo con caída & Positivo \\
Fuckuda (unterberger) & Positivo (+++) & Positivo (+) \\
Marcha: & & Normal \\
\multicolumn{1}{c}{ Longitud del paso } & Disminuida & Normal \\
\multicolumn{1}{c}{ Velocidad del paso } & Disminuida & Normal \\
\hline
\end{tabular}


derecho e hipoacusia derecha progresiva. Fue diagnosticada de EM en base a la electrococleografía.

A pesar de haber recibido diferentes tratamientos médicos, incluyendo la administración de gentamicina intratimpánica no mostraba mejoría, por lo que hace 12 años fue intervenida con cirugía de derivación del saco endolinfático con éxito y desaparición de los vértigos rotatorios. Los potenciales evocados auditivos, realizados hace 5 años, registraron sordera neurosensorial.

Dos años antes de su evaluación se instauró un cuadro de desequilibrio que se exacerbaba con los cambios posturales, siendo mayor al girar a la derecha; marcha con amplia base de sustentación y vértigo posicional, que se tornaron permanentes. Recibió tratamiento farmacológico, pero los síntomas persistieron, cada vez con mayor inestabilidad. La paciente refería limitación marcada para realizar sus actividades de vida diaria, con incapacidad para el trabajo y ausentismo laboral frecuente, necesitando ser atendida por urgencia en reiteradas ocasiones, requiriendo incluso ser hospitalizada.

Hace un año fue intervenida con RV, encontrándose en la evaluación pruebas cerebelosas normales, reflejo vestíbulo ocular (VOR) alterado a la derecha en el test de impulso cefálico y en la sacudida cefálica, nistagmus posicional provocado (Prueba de Dix Hallpike) positivo para el canal posterior derecho, la prueba de Romberg positivo hacia la derecha y Unterberger positivo.

De acuerdo a la evaluación, se le realizó la maniobra de Epley para la reposición otolítica y ejercicios de adaptación y sustitución (con el uso de estímulos visuales mediante imágenes, para la interacción visualvestibular, así mejorar la estabilización de la mirada), ejercicios de habituación, del equilibrio vestibular y de control postural para prevención de caídas.
La intervención con RV fue en una sola sesión, posteriormente realizó terapia domiciliaria por dos meses según las pautas indicadas, fue reevaluada al término del mismo en los aspectos clínicos (Tabla 1) y funcionales (Tabla 2). Como tratamiento de mantenimiento, se le proporcionó una cartilla con pautas de ejercicios vestibulares.

En la actualidad, la paciente conserva el estado funcional logrado a los dos meses post intervención, y no necesita medicamentos ni atenciones de salud por este mismo motivo. De manera eventual presenta síntomas vestibulares leves, que mejoran al realizar los ejercicios vestibulares.

\section{DISCUSIÓN}

En el estadio tardío de la EM, los episodios de vértigo disminuyen y se va instaurando el desequilibrio permanente. Una vez que se controlan los síntomas agudos fluctuantes de la EM, se recomienda la RV, que de manera efectiva mejoran el equilibrio y la funcionalidad (2).

El reporte de este caso fue realizado para mostrar los efectos de la RV en una paciente con persistencia de vértigo posicional y desequilibrio moderado por EM en su estadio tardío.

En relación al cuadro clínico de la etapa crónica, la paciente desarrolló episodios vertiginosos tipo VPPB, asociación que se muestra en el estudio de Taura et al (3). Estos síntomas generaron gran discapacidad en la paciente, conforme lo enfatiza el estudio de Gutierrez et al (9).

Existen diversos tratamientos para la EM, tales como dietéticos (10), farmacológicos $(11,12)$, inyecciones intratimpánicas $(13,14)$, quirúrgicos $(12,15)$ y la RV que está demostrada ser una alternativa eficaz en el estadio tardío de la EM $(2,6)$.

Tabla 2. Resultados de las actividades funcionales con intervención con rehabilitación vestibular.

\begin{tabular}{ll}
\hline $\begin{array}{l}\text { Antes de la intervención con Rehabilitación } \\
\text { Vestibular }\end{array}$ & $\begin{array}{l}\text { Después de dos meses de la intervención con } \\
\text { Rehabilitación Vestibular }\end{array}$ \\
\hline Gran limitación de las actividades de vida diaria & Realiza con normalidad las actividades de vida diaria \\
Moderada limitación del desempeño laboral & Sin limitación del desempeño laboral \\
Gran ausentismo laboral & Mínimo ausentismo laboral \\
Gran deterioro de la Calidad de vida & Nivel aceptable de calidad de vida \\
\hline
\end{tabular}


En un primer momento, a la paciente se le realizó la maniobra de reposición, en relación a un resultado positivo del test posicional de Dix- Hallpike, el que contribuyó de forma significativa con la disminución del vértigo posicional, como lo demuestran los estudios de Monobe et al. (16), y el de Aburto et al. (17), que realizaron la maniobra de Epley con una tasa de éxito del 90,3\% y 92\% respectivamente.

Posteriormente por la persistencia de la oscilopsia, se realizaron ejercicios de compensación del VOR, a través de estímulos visuales mediante imágenes (estáticas y en movimiento), similar a los resultados del estudio de Manso et al., en Sao Paulo (18); asimismo, se estimuló el reflejo vestíbulo espinal y se provocó situaciones de inestabilidad, de modo que la paciente desarrolle estrategias compensatorias para lograr menor oscilación, el que fué confirmado al evaluar la prueba de Romberg.

Actualmente existen otras estrategias de RV, como la utilización de realidad virtual en la EM (19), demostrándose que la intervención con estímulos visuales digitales es más eficaz frente a los estímulos visuales no digitales en la mejoría de la función vestibular y del equilibrio corporal, por lo que podría ser una herramienta útil para una mejor intervención con RV (18).

Los resultados de la intervención con terapia física vestibular asociado a tratamiento médico no invasivo en el control del vértigo, es similar a la aplicación de gentamicina intratimpánica; por lo que en este caso, podría haberse intervenido de forma precoz con RV (6).

El cuadro clínico y la calidad de vida en los aspectos físicos, emocionales y funcionales, mejoraron de manera significativa en la paciente, como lo demuestran las investigaciones (20); de forma semejante el estudio de Kazuko et al., demostró su eficiencia al comparar los resultados del "Dizziness Handicap Inventory" pre y post RV en pacientes con vértigo crónico (21), y el estudio de Riveros et al., que considera que la RV es una terapia efectiva y que existe una alta probabilidad de que mejore además su estado físico y mental (22).

La RV realizada en la paciente fue personalizada, la que se demuestra ser más efectiva y exitosa, como lo señalan los estudios en el que utilizan protocolos de tratamiento diferentes, de acuerdo a la evaluación (23); además, los efectos de la RV tanto convencional como multimodal en la capacidad funcional y el control del equilibrio, son de fácil aplicación y requieren recursos mínimos, pudiendo ser aplicable en diversos entornos terapéuticos (24).

El resultado de la RV en la paciente fue favorable; sin embargo, no se puede llegar a conclusiones con un solo caso. Con la acumulación de más casos de pacientes con EM, se podría hacer investigaciones de los efectos de la RV en las diferentes fases de la enfermedad. Además, cabe señalar la importancia del abordaje integral en el tratamiento de pacientes con EM.

Diversas investigaciones en curso están aportando gran información sobre los efectos beneficiosos de la RV, y de los mecanismos a través de los cuales se consigue el control del vértigo y la inestabilidad; sin embargo, el impacto negativo que provoca la EM y las escasas publicaciones en RV de la EM; refuerza la necesidad de ampliar los conocimientos en el tema.

\section{Declaración de conflicto de interés:}

Los autores declaran no tener conflictos de intereses

\section{Contribución de autoría:}

YPE; MJP, JJL; LRG; RTE: concepción, recolección de datos, discusión y redacción del artículo.

\section{Correspondencia:}

Yuly Rossana Polo Espinoza

Calle Los Quechuas 172. Cooperativa Andahuaylas 1era Etapa. Santa Anita. Lima, Perú

Correo electrónico: rossana.polo@gmail.com

\section{REFERENCIAS BIBLIOGRÁFICAS}

1. Micheli F, Nogues M, Asconape J, Fernández M, Biller J. Tratado de Neurología Clínica. Madrid: Editorial Médica Panamerica; 2003.

2. Pérez H, López J, Morales C, Pérez N. Enfermedad de Menière: desde las ciencias básicas hacia la medicina clínica Badalona: Ediciones Médicas EUROMEDICE; 2009.

3. Taura A, Funabiki K, Ohgita H, et al. One-third of vertiginous episodes during the follow-up period are caused by benign paroxysmal positional vertigo in patients with Meniere's disease. Acta Otolaryngol. 2014; 134(11):1140-5. doi: 10.3109/00016489.2014.936624

4. Sauvage J. Vértigos: Manual de diagnóstico y rehabilitación. Madrid: Elsevier; 2017. 
5. Havia M, Kentala E, Pyykkö I. Prevalence of Menière's disease in general population of Southern Finland. Otolaryngol Head Neck Surg 2005; 133(5): 762-768. Doi: http://journals.sagepub.com/ doi/10.1016/j.otohns.2005.06.015

6. Gottshall K, Hoffer M, Moore R, BaloughB. The role of vestibular rehabilitation in the treatment of Meniere's Disease. Otolaryngol Head Neck Surg. 2005; 133(3):326-8. Doi: https://doi.org/10.1016/j. otohns.2005.06.001

7. Herdman S. Vestibular rehabilitation. Philadeldhia: FA Davis Comp; 2007.

8. Stokes M, Stack E. Fisioterapia en la rehabilitación neurológica. Madrid: Elsevier; 2013.

9. Gutiérrez-Márquez A, Jáuregui-Renaud K, ViverosRenteria L, Villanueva-Padrón L. Discapacidad por enfermedad auditiva y vestibular en un centro de atención especializada. Gac Méd Méx. 2005; 141(2): 105-110. (Citado 19 de enero de 2018) Disponible en: http://www.scielo.org.mx/scielo.php?script=sci arttext\&pid=S0016-38132005000200004\&lng=es

10. Miyashita T, Inamoto R, Fukuda S, et al. Hormonal changes following a low-salt diet in patients with Ménière's disease. Auris Nasus Larynx. 2017; 44(1):52-57. doi: 10.1016/j.anl.2016.03.001

11. Teggi R, Gatti O, Sykopetrites V, Quaglieri S, Benazzo M, Bussi M. Association of cinnarizine and betahistine in prophylactic therapy for Ménière's disease with and without migraine. Acta Otorhinolaryngol Ital. 2014; 34(5):349-353.

12. Rivas J, Ariza H. Tratado de otología y audiología. Bogotá: AMOLCA; 2007.

13. Ren H, Yin T, Lu Y, Kong W, Ren J. Intratympanic dexamethasone injections for refractory Meniere' $\mathrm{s}$ disease. Int J Clin Exp Med. 2015; 8(4):6016-6023.

14. Riveros H, Callejas C, Fernández F, Cohen M. Experiencia en el tratamiento de la enfermedad de Ménière con terapia transtimpánica. Rev otorrinolaringol cir cabeza cuello. 2005; 65(3): 187192. (Citado 19 de enero de 2018) Disponible en: http://www.sochiorl.cl/uploads/05(22).pdf

15. Cruz F, Aquino J, Torres T. Decompression of the Endolymphatic Sac: Vertigo and Hearing Analysis. Int. Arch. Otorhinolaryngol. 2009; 13(4):426-430. (Citado 19 de enero de 2018) Disponible en: http:// www.arquivosdeorl.org.br/conteudo/acervo_port. asp? id $=654$

16. Monobe H, Sugasawa K, Murofushi T. The outcome of the canalith repositioning procedure for benign paroxysmal positional vertigo: are there any characteristic features of treatment failure cases? Acta Otolaryngol Suppl. 2001; 545:38-40.
17. Aburto R, Medrano ML, De la Torre J. Rehabilitación Vestibular en el VPPB. Rev otorrinolaringol Cir cabeza cuello. 2002; 62(2):123-128.

18. Manso A, Ganança M, Caovilla H. Vestibular rehabilitationwithvisualstimuliinperipheralvestibular disorders. Braz j otorhinolaryngol. 2016; 82(2):232241. (Citado 12 de noviembre de 2017) Disponible en: $\quad$ http://www.scielo.br/scielo.php?script=sci arttext\&pid=S1808-86942016000200232\&lng=en

19. Garcia A, Ganança M, Cusin F, Tomaz A, Ganança F, Caovilla H. Reabilitação vestibular comrealidade virtual na doença de Ménière. Braz $\mathrm{J}$ otorhinolaryngol. 2013; 79(3):366-374. doi: http:// dx.doi.org/10.5935/1808-8694.20130064

20. Soares S, Gonçalves M, Teixeira C, Romualdo P, Santos J. Influência da reabilitação vestibular na qualidade de vida de indivíduos labirintopatas. Rev CEFAC. 2014; 16(3): 732-738. doi: http:// dx.doi.org/10.1590/1982-0216201418211

21. Nishino LK, Granato L, Campos CAH. Quality of life questionnaire application in patients before and after vestibular rehabilitation. Int. Arch. Otorhinolaryngol. 2008; 12(4): 517- 522. (Citado 12 de noviembre de 2017) Disponible en: http://www. arquivosdeorl.org.br/conteudo/acervo_port. asp?id $=566$

22. Riveros H, Correa C, Anabalón JL, Aranís C. Efectividad de la rehabilitación vestibular en una serie clínica. Rev Otorrinolaringol. Cir. Cabeza Cuello. 2007; 67(3): 229-236. doi: http://dx.doi. org/10.4067/S0718-48162007000300004

23. Nishino LK, Ganança C, Manso A, Campos CAH de, Korn G. Reabilitação vestibular personalizada: levantamento de prontuários dos pacientes atendidos no ambulatório de otoneurologia da I.S.C.M.S.P. Rev Bras Otorrinolaringol. 2005; 71(4): 440-447. Doi: ht t p://dx.doi .org/10.1590/S003472992005000400007

24. Ricci NA, Aratani MC, Caovilla HH, Ganança FF. Effects of conventional versus multimodal vestibular rehabilitation on functional capacity and balance control in older people with chronic dizziness from vestibular disorders: design of a randomized clinical trial. Trials. 2012; 13:246. doi: 10.1186/1745-6215$13-246$

Recibido: 16/05/2018

Aceptado: 28/06/2018 